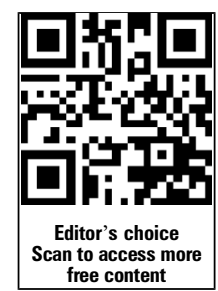

For numbered affiliations see end of article.

\section{Correspondence to}

Professor Dr Georg Hansmann, Department of Pediatric Cardiology and Critical Care, Hannover Medical School, Carl-Neuberg-Str. 1, Hannover 30625, Germany; hansmann. georg@mh-hannover.de, georg.hansmann@gmail.com

LC, EL and LG contributed equally.

Received 5 July 2015 Revised 31 October 2015 Accepted 6 November 2015

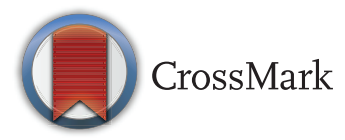

To cite: Calvier $\mathrm{L}$, Legchenko $E$, Grimm L, et al. Heart 2016;102: 390-396.

\title{
Galectin-3 and aldosterone as potential tandem biomarkers in pulmonary arterial hypertension
}

\author{
Laurent Calvier, ${ }^{1}$ Ekaterina Legchenko, ${ }^{1}$ Lena Grimm, ${ }^{1}$ Hannes Sallmon, ${ }^{2}$ \\ Adam Hatch, ${ }^{3}$ Brian D Plouffe, ${ }^{3}$ Christoph Schroeder, ${ }^{4}$ Johann Bauersachs, ${ }^{5}$ \\ Shashi K Murthy, ${ }^{3,6}$ Georg Hansmann ${ }^{1}$
}

\begin{abstract}
Background Several studies have identified circulating biomarkers to be associated with the presence and severity of pulmonary arterial hypertension (PAH). Recent evidence supports a role for galectin-3 (Gal-3) and the mineralcorticoid aldosterone in left ventricular failure. However, studies on aldosterone together with Gal-3 in PAH are lacking.
\end{abstract}

Objective We investigated a novel Aldosterone-galectin3 (Gal-3) tandem and several other potential PAH biomarkers and their association with the disease severity. Methods A total of 57 patients, 41 with idiopathic PAH. (IPAH) and 16 with PAH associated with connective tissue disease (CTD), and 8 age-matched, non-relative controls were studied. Gal-3, aldosterone and other potential protein plasma concentrations were measured by single ELISA and multi-array MSD (Meso Scale Discovery) technology.

Results Gal-3 values were increased in both patients with IPAH $(12.2 \pm 0.6 \mathrm{ng} / \mathrm{mL} ; \mathrm{p}<0.05)$ and with PAH-CTD $(14.1$ $\pm 1.6 \mathrm{ng} / \mathrm{mL} ; p<0.05)$ versus control $(8.5 \pm 0.9 \mathrm{ng} / \mathrm{mL})$, while aldosterone was significantly elevated in IPAH only (248.5 $\pm 38.8 \mathrm{pg} / \mathrm{mL}$ vs control $71.9 \pm 18.2 \mathrm{pg} / \mathrm{mL} ; \mathrm{p}<0.05)$. In addition, aldosterone, Gal-3, and N-terminal pro-brain natriuretic peptide (NT-proBNP) values were all higher in patients in WHO functional class II-III versus PAH functional class I or controls. The vascular injury marker intercellular adhesion molecule 1 (ICAM-1) was increased in IPAH and PAH-CTD versus controls $(559.5 \pm 18.2 \mathrm{pg} / \mathrm{mL}$ and 734.1 $\pm 59.4 \mathrm{pg} / \mathrm{mL}$ vs controls $394.8 \pm 39.3 \mathrm{pg} / \mathrm{mL}, \mathrm{p}<0.05$, $p<0.0001$, respectively), whereas vascular cell adhesion molecule 1 (VCAM-1) and proinflammatory, anti-angiogenic interleukin-12 (IL-12) were elevated in PAH-CTD only $(879.5 \pm 110.0 \mathrm{pg} / \mathrm{mL}$ and $391.2 \pm 70.3 \mathrm{pg} / \mathrm{mL}$ vs controls $489.8 \pm 44.6 \mathrm{pg} / \mathrm{mL}, \mathrm{p}<0.01$, and $102.1 \pm 15.2 \mathrm{pg} / \mathrm{mL}$, $p<0.01$, respectively).

Conclusions Heightened Gal-3 and aldosterone plasma concentrations in PAH patients indicate a role for Gal-3 signalling in the pathobiology of IPAH and PAH-CTD, and may serve as biomarkers for functional status and progression of disease.

\section{INTRODUCTION}

Pulmonary arterial hypertension (PAH) and associated heart failure is a complex, multifactorial disease with a poor prognosis. ${ }^{1-3}$ None of the current therapies has been shown to be universally effective, nor have these therapies been able to reverse advanced pulmonary vascular disease or to prevent right heart failure in advanced $\mathrm{PAH} .{ }^{2}{ }^{3}$ The progressive nature and heterogeneous aetiology of
PAH underlines the critical need for both early diagnosis and accurate stratification to allow tailored, efficient therapies. ${ }^{4}$

Several studies have identified circulating biomarkers associated with PAH, including markers of endothelial dysfunction, vascular injury and remodelling, myocardial damage, inflammation, and oxidative stress. N-terminal pro-brain natriuretic peptide (NT-proBNP), endothelin-1, interleukin-6, interferon- $\alpha, \mathrm{C}$ reactive protein (CRP), serotonin, as well as endothelial progenitor cells, and recently identified pulmonary hypertension ( $\mathrm{PH}$ )-relevant microRNAs, belong to the most validated markers. ${ }^{56}$

A role for aldosterone in pulmonary vascular remodelling and fibrosis has been suggested by increased plasma and tissue concentrations of aldosterone in experimental models, and also in patients with PAH. ${ }^{7-10}$ Aldosterone contributes to the activation of pathways that decrease bioavailable nitric oxide levels, induce inflammation, and promote cell proliferation, migration, extracellular matrix remodelling, and fibrosis. Moreover, clinical mineralocorticoid receptor (MR) blockade in addition to endothelin-1 receptor inhibition has been associated with a better prognosis in clinical PAH. ${ }^{11}$

The molecular mechanisms by which aldosterone exerts its cardiovascular effects are complex. ${ }^{12}$ Recent evidence indicates a critical role for galectin-3 (Gal-3), a $\beta$-galactoside-binding lectin, in inflammation, fibrosis, and heart failure, downstream of aldosterone. ${ }^{13} 14$ Accordingly, Gal-3 is already established as a biomarker for cardiac fibrosis, and chronic left ventricular heart failure. ${ }^{15} 16$ Consequently, Gal-3 has been approved by the US Food and Drug Administration as a new biomarker for additive risk stratification in heart failure, and received a class IIb recommendation in the most recent American Heart Association/American College of Cardiology (AHA/ACC) guidelines. ${ }^{17}$

However, to date, combined clinical studies on the role of both Gal-3 and aldosterone in PAH are lacking. Here, we investigate several biomarkers in $\mathrm{PAH}$ associated with vascular injury and myocardial fibrosis, and identify Gal-3 and aldosterone as potential tandem biomarkers in patients with idiopathic PAH (IPAH) or PAH associated with connective tissue disease (PAH-CTD).

\section{METHODS \\ Study population}

During the research conferences of the Pulmonary Hypertension Association in California (2010) and 
Florida (2012), 57 patients (IPAH, n=41; PAH-CTD, $n=16$ ) and eight healthy, age- and gender-matched, non-relative controls were recruited. Exclusion criteria were male sex, sleep apnoea, liver disease, chronic obstructive pulmonary disease (COPD), and pulmonary fibrosis. All participants completed a questionnaire regarding their demographic data (tables 1 and 2) and medication (table 2). Written informed consent was obtained from each subject. The study has been approved by the internal review board of Northeastern University (IRB \# 11-07-19). Detailed characteristics of each individual PAH patient can be found in table 2 .

\section{Biomarker assays}

EDTA whole blood samples were collected through peripheral venipuncture in non-fasting subjects, processed to plasma (ie, immediate centrifugation for $10 \mathrm{~min}$ at $1300 \mathrm{~g}$ ), and stored at $-80^{\circ} \mathrm{C}$. Gal-3 and aldosterone concentrations were measured in one thaw step by BGM Gal-3 ELISA (BG Medicine, Waltham, Massachusetts, USA) and by aldosterone ELISA (RE52301, IBL International, Hamburg, Germany), respectively, according to the manufacturers' instructions. For assessment of NT-proBNP, the singleplex sandwich electrochemiluminescent immunoassay by Meso Scale Discovery was used, according to the manufacturer's protocol. All other molecules were measured using Meso Scale Discovery's Multi-Array technology (MSD, Angiogenesis Panel 1 Human, Cytokine Panel 1 Human, Proinflammatory Panel 1 Human, Vascular Injury Panel 2 Human). Signal intensities were measured with the MESO QuickPlex SQ 120 instrument (Meso Scale Discovery, Rockville, Maryland, USA) and analysis was performed using the Discovery Workbench software V.4.0 (Meso Scale Discovery, Rockville, Maryland, USA), according to the manufacturer's instructions. All protein markers of which more than $50 \%$ of the measurements fell into the detection range of the standard curves were considered valid.

\section{Statistical analysis}

Statistical analyses were performed with GraphPad Prism V.6 software. Data are expressed as mean \pm SEM or median values with IQR as appropriate. Data were tested for normal distribution with D'Agostino-Pearson omnibus, Shapiro-Wilk, and Kolmogorov-Smirnov tests. For comparison of data, one-way

\begin{tabular}{|c|c|c|c|c|}
\hline & Control & PAH (total) & IPAH & CTD \\
\hline$N$ & 8 & 57 & 41 & 16 \\
\hline Age (years) & $49.3(33-67)$ & $47.2(19-77)$ & 44.6 (19-74) & $53.8(28-77)$ \\
\hline Male sex (n) & 0 & 0 & 0 & 0 \\
\hline Height (m) & 1.69 & 1.63 & 1.63 & 1.62 \\
\hline Weight (kg) & 73.7 & 70.3 & 69.0 & 73.5 \\
\hline BMI $\left(\mathrm{kg} / \mathrm{m}^{2}\right)$ & 25.8 & 26.5 & 25.9 & 27.0 \\
\hline \multicolumn{5}{|c|}{ Race/ethnicity } \\
\hline White & 7 & 32 & 23 & 9 \\
\hline Black & 0 & 2 & 0 & 2 \\
\hline Asian & 1 & 1 & 0 & 1 \\
\hline Hispanic & 0 & 5 & 5 & 0 \\
\hline Other & 0 & 17 & 13 & 4 \\
\hline
\end{tabular}

Values represent the number of subjects or mean values, as appropriate. Control subjects were age and gender matched to study subjects.

$\mathrm{BMI}$, body mass index; CTD, pulmonary arterial hypertension associated with connective tissue disease; IPAH, idiopathic pulmonary arterial hypertension; PAH, pulmonary arterial hypertension. analysis of variance (ANOVA) or Kruskal-Wallis tests were used, corrected for multiple comparisons by Dunn's test, as appropriate. Values of $\mathrm{p}<0.05$ were considered significant.

\section{RESULTS}

\section{Demographic characteristics}

Demographic characteristics of the study subjects by groups are listed in table 1. Detailed information on each individual $\mathrm{PAH}$ patient including age, weight, body mass index, $\mathrm{PAH}$ diagnosis (IPAH, PAH-CTD), WHO functional class (FC) and medication is provided in table 2 .

\section{Plasma concentrations of Gal-3, aldosterone and NT-proBNP in PAH patients}

In order to test whether Gal-3, aldosterone and NT-proBNP plasma concentrations were increased in patients with $\mathrm{PAH}$ versus controls, ELISAs were performed in control $(n=8)$, IPAH $(n=41)$, and CTD $(n=16)$ plasma. Aldosterone plasma concentrations were elevated only in IPAH $(248.5 \pm 38.8 \mathrm{pg} / \mathrm{mL}$ vs control $71.9 \pm 18.2 \mathrm{pg} / \mathrm{mL} ; \mathrm{p}<0.05)$, while the difference between controls and PAH-CTD patients $(147.8 \pm 28.7 \mathrm{pg} / \mathrm{mL}$ vs control $71.9 \pm 18.2 \mathrm{pg} / \mathrm{mL}$ ) did not reach statistical significance (figure 1A, B). Gal-3 concentrations were significantly increased in patients with IPAH $(12.2 \pm 0.6 \mathrm{ng} / \mathrm{mL})$ and PAH-CTD $(14.1 \pm 1.6 \mathrm{ng} / \mathrm{mL})$ versus controls $(8.5 \pm 0.9 \mathrm{ng} / \mathrm{mL} ; \mathrm{p}<0.05$; figure $1 \mathrm{C}, \mathrm{D})$. In addition, NT-proBNP values were increased in both IPAH and PAH-CTD patients as compared to controls $(1575 \pm 291 \mathrm{ng} / \mathrm{mL} ; \mathrm{p}<0.05 ;$ and $1544 \pm 380 \mathrm{ng} / \mathrm{mL} ; \mathrm{p}<0.05$, respectively; figure $1 \mathrm{E}, \mathrm{F})$.

Subsequently, we evaluated whether plasma concentrations of aldosterone, Gal-3, and NT-proBNP differed between PAH patients, grouped by WHO FC (FC I, n=10; FC II-III, n=53). Only one patient fulfilled the criteria for WHO FC IV and was, therefore, excluded from the analysis. Aldosterone, Gal-3, and NT-proBNP concentrations were all significantly increased in FC II-III PAH versus controls. A significant difference between FC II-III and FC I PAH was found only for Gal-3 and NT-proBNP but not for aldosterone plasma concentrations (figure 2A-B, C-D, E-F, respectively).

\section{Other markers of vascular injury and inflammation}

For our analysis we used several panels of the Meso Scale Discovery's Multi-Array assays in control $(n=5)$, IPAH $(n=41)$, and CTD $(n=16)$ plasma. Quantitative measurements were considered reliable when more than $50 \%$ of the measurements fell within the linear range of the respective standard curves. Such markers included intercellular adhesion molecule 1 (ICAM-1), vascular cell adhesion molecule 1 (VCAM-1), serum amyloid A (SAA), CRP, interleukin-12 (IL-12), fibroblast growth factor 2 (FGF-2) (figure 3), as well as interleukin-16 (IL-16), vascular endothelial growth factor (VEGF), VEGF-C, and VEGF-D (figure 4). Plasma ICAM-1 concentrations were increased in patients with IPAH and PAH-CTD versus controls $(559.5 \pm 18.2$ $\mathrm{pg} / \mathrm{mL}$ and $734.1 \pm 59.4 \mathrm{pg} / \mathrm{mL}$ vs controls $394.8 \pm 39.3 \mathrm{pg} / \mathrm{mL}$, $\mathrm{p}<0.05, \mathrm{p}<0.0001$, respectively). Of note, the average plasma ICAM-1 concentration was significantly higher in PAH-CTD than in IPAH patients $(\mathrm{p}<0.01$; figure $3 \mathrm{~A})$. In addition, VCAM-1 and IL-12 values were elevated in PAH-CTD patients versus controls $(879.5 \pm 110.0 \mathrm{pg} / \mathrm{mL}$ and $391.2 \pm 70.3 \mathrm{pg} / \mathrm{mL}$ vs controls $489.8 \pm 44.6 \mathrm{pg} / \mathrm{mL}, \mathrm{p}<0.01$, and $102.1 \pm 15.2 \mathrm{pg} /$ $\mathrm{mL}, \mathrm{p}<0.01$, respectively; figure $3 \mathrm{~B}, \mathrm{E})$, but not in IPAH patients. The vascular injury/inflammatory markers SAA and CRP plasma concentrations tended to be higher in IPAH and PAH-CTD versus controls (ns; figure 3C, D). FGF-2, that has 
Table 2 Individual PAH patient characteristics, including medication.

\begin{tabular}{|c|c|c|c|c|}
\hline Age (yrs) & Weight (kg) & BMI $\left(\mathrm{kg} / \mathrm{m}^{2}\right)$ & WHO Class & Medication* \\
\hline \multicolumn{5}{|c|}{ Diagnosist: IPAH } \\
\hline 63 & 59 & 26.2 & 1 & SIL, TREPIN, VAL, 02 \\
\hline 31 & 70 & 28.4 & 1 & AMB, SIL, TREPIN, DIG, 02 \\
\hline 33 & 64 & 22.7 & 1 & NIF, WAR \\
\hline 33 & 57 & 20 & 1 & SIL, WAR, PPI \\
\hline 33 & 91 & 28,1 & 1 & THY, PRE, TREPIV, 02, intravenous immunoglobuline, IRO, WAR, VEN, AMI, SPI, LIS, TOR, BOS, PPI, TRAM, PRC \\
\hline 49 & 59 & 21,7 & 1 & BOS, FUR, NIF, WAR, SAL, SER \\
\hline 40 & 94 & 34,5 & 1 & AMB, AML, FUR, TAD \\
\hline 26 & 58 & 19,4 & 1 & AML, FUR, SIL, WAR \\
\hline 41 & 59 & 20.9 & 2 & BOS, SIL, NIF, WAR \\
\hline 50 & 49 & 19.9 & 2 & BOS, TAD, ASA, SIM, SER \\
\hline 47 & 70 & 28.4 & 2 & BOS, TREPIV, HCT, WAR, ESC, GAB \\
\hline 19 & 54 & 21.9 & 2 & SIL, TREPIV, FUR, ALD, DIG, WAR, IRO, PPI \\
\hline 74 & 84 & 29.8 & 2 & SIL, THY, WAR, SD, CEL \\
\hline 50 & 102 & 36.1 & 2 & BOS, SIL, FUR, ALD, WAR, FLU, FEX, PPI \\
\hline 60 & 75 & 31.2 & 2 & AMB, TRI, ALD, NAP \\
\hline 44 & 61 & 23.8 & 2 & EPIV, ALD, FLU, BUP, GAB \\
\hline 38 & 78 & 26.1 & 2 & BOS, TAD, TREPIV, FUR \\
\hline 49 & 86 & 30.5 & 2 & AMB, AML, TREPIN, TOR, HCT, PRA, PPI, 02 \\
\hline 31 & 68 & 22.2 & 2 & PGI, WAR, ESC, GAB, PPI \\
\hline 34 & 51 & 22.7 & 2 & BOS, SIL, TREPSC, FUR, DIG, IRO, HYD, PRE \\
\hline 48 & 68 & 25 & 2 & AMB, TAD, TREPPO, CAV, WAR, BUM, RIS, PPI, TRAM, HYD, Calcium, CET, TEM, TRA, ALP \\
\hline 43 & 88 & 35 & 2 & BOS, SIL, SPI, FUR, ATO, WAR, ILO \\
\hline 68 & 53 & 19,5 & 2 & NIF, WAR, SIM, FLU, PPI, ASA, ALP, IRO, PRC, COD, RAN \\
\hline 52 & 71 & 27,4 & 2 & BOS, TAD, SPI, FUR, MEF, SIT, LIS, DIG, CLG, DUL, LOV, THY, KCL, FAM \\
\hline 37 & 109 & 33,6 & 2 & TAD, AMB, WAR, CET, RAN, SER, GUA, FLT \\
\hline 48 & 66 & 24,8 & 2 & TREPIN, BOS, DIG, TAD, DIL \\
\hline 21 & 61 & 23 & 2 & SIL, AMB, WAR, DIG, PPI, FUR, SPI, Depo Provera \\
\hline 31 & 56 & 21,9 & 2 & SIL, AMB, TREPPO, PPI, TOP, SOL, DIG \\
\hline 65 & 51 & 24,6 & 2 & NIF, THY, SIL, VYT \\
\hline 60 & 66 & 24,8 & 2 & TREP, AMB, TAD, GAB, DIG, SPI, SER \\
\hline 39 & 64 & 27,7 & 2 & TREP, BOS, FUR, PPI, SPI \\
\hline 29 & 56 & 19,4 & 2 & AMB, TAD, TOP, DAB, FUR, IRO \\
\hline 44 & 38 & 14 & 2 & FSA, AMT, AZE, WAR, DIG, FUR, HCT, PPI, MTZ, CIC, 02, PRM, TREPIV, SIL, SPI, TRAM, TRC, LEV \\
\hline 69 & 75 & 28,2 & 2 & AMB, DIL, CLO, LIS, MEF, PIO, FUR, RAN, ROS, SIT, AMT, REP, DAB, MET \\
\hline 45 & 52 & 20,3 & 2 & TREPIN, BOS, FUR, WAR \\
\hline 32 & 88 & 34,4 & 2 & PRC, BOS, Calcium, IMB, Immodium, LOR, 02, RAN, THY, TREPIV \\
\hline 65 & 84 & 27,4 & 2 & DIL, RAL, RAN, FLT, BOS, SPI, Finacea, ASA, CLB, CIT, BME, TREPIN \\
\hline 44 & 54 & 19,8 & 3 & SIL, AMB, IMB, EPIV, Calcium, THY, TOR, PPI, DIL, MON, CET, SPI \\
\hline 38 & 70 & 28,4 & 3 & BOS, WAR, NOR, ARG, AML, HCT, SD, MOM \\
\hline 48 & 86 & 37,2 & 3 & EPIV, BOS, TAD, FUR, PPI, IRO, FA \\
\hline 59 & 86 & 31.6 & 3 & AMB, SIL, TREPIN, FUR, ALD, WAR, SIM, IRO, OM3, PPI \\
\hline \multicolumn{5}{|c|}{ Diagnosis*: CTD } \\
\hline 50 & 107 & 37.9 & 2 & BOS, SIL, DIL, FUR, WAR, 02 \\
\hline 62 & 64 & 26.3 & 2 & SIL, MTX, PPI \\
\hline 77 & 72 & 27.1 & 2 & BOS, NIF, HCT, THY, PRED, MTX, OM3 \\
\hline 58 & 70 & 27.3 & 2 & TAD, HCT, WAR, IRO \\
\hline 50 & 60 & 26.0 & 2 & AMB, AML, FUR, ALD, DIG, ALL, COL, URS, 02 \\
\hline 51 & 54 & 23.4 & 2 & BOS, SIL, DIL, THY, PRED \\
\hline 52 & 104 & 38.2 & 2 & BOS, SIL, TREPIN, FUR, BUM, THY, INS, DUL, PRE \\
\hline 57 & 84 & 30.9 & 2 & NIF, TREPIV, MTX, PRED, FA, PPI \\
\hline 46 & 91 & 36,9 & 2 & SIL, QUE, WAR, TREPIN, PPI, HXC, LOZ \\
\hline 60 & 68 & 22,7 & 2 & TREPIN, AMB, SIL, FUR \\
\hline 57 & 52 & 20,3 & 2 & AMB, FUR, BUP, CIT, PPI, PEN, ZOL, FEX \\
\hline 57 & 64 & 26,0 & 2 & BOS, TAD, FUR, SPI, 02 \\
\hline 40 & 82 & 26.8 & 3 & BOS, SIL, AML, TREPIV, FUR, ALD, PRED, LOS, CLO, ATE \\
\hline 66 & 83 & 31.2 & 3 & BOS, SIL, FUR, ALD, THY, LIS, PRED, AZA, PPI, PAR, 02 \\
\hline 28 & 58 & 21,8 & 2 & TREPIV, SIL, WAR, HXC, FUR, PRC/OXC, MMF, FLU, THY, PRED, FA, PPI, RAN, ZOL \\
\hline 50 & 63 & 25,2 & 4 & PPI, DOM, SIL, AMB, FUR, AML, FA, PRE, LOR \\
\hline
\end{tabular}

* Medications (prostanoids are in bold): $\mathrm{ALD}=$ aldactone $\mathrm{PO} ; \mathrm{ALL}=$ allopurinol $\mathrm{PO} ; \mathrm{AMB}=$ ambrisentan $\mathrm{PO} ; \mathrm{AML}=$ amlodipine $\mathrm{PO} ; \mathrm{ALP}=$ alprazolam $\mathrm{PO} ; \mathrm{AMI}=$ amiloride $\mathrm{PO}$;

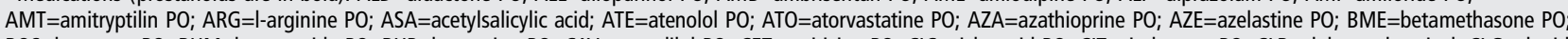
$\mathrm{BOS}=$ bosentan $\mathrm{PO} ; \mathrm{BUM}=$ bumetanide $\mathrm{PO} ; \mathrm{BUP}=$ bupropion $\mathrm{PO} ; \mathrm{CAV}=$ carvedilol $\mathrm{PO} ; \mathrm{CET}=$ cetirizine $\mathrm{PO} ; \mathrm{CIC}=$ ciclenosid $\mathrm{PO} ; \mathrm{CIT}=\mathrm{citalopram} \mathrm{PO} ; \mathrm{CLB}=$ clobetasol topical; $\mathrm{CLG}=\mathrm{clopidogrel}$ PO; $C L O=$ clonidine PO; $C O D=$ codein PO; $C O L=$ colchicin PO; $\mathrm{DAB}=$ dabigatranexilat PO; DIG=digoxin PO; DIL=diltiazem; DIP=dipyridamole; $\mathrm{DOM}=$ domperidone PO; $\mathrm{DUL}=$ duloxetine PO; EPIV=epoprostenol IV; ESC=escitalopram PO; FA=folic acid PO; FAM=famotidin PO; FEX=fexofenadine PO; FLT=fluticasone INH; FLU=fluoxetin PO; FSA=fluticasone/salmeterol INH; $\mathrm{FUR}=$ furosemide $\mathrm{PO} ; \mathrm{GAB}=$ gabapentin $\mathrm{PO} ; \mathrm{GUA}=$ guifenesin $\mathrm{PO} ; \mathrm{HCT}=$ hydrochlorothiazide $\mathrm{PO} ; \mathrm{HXC}=$ hydroxychloroquine $\mathrm{PO} ; \mathrm{HYD}=$ hydrocodone; ILO=iloprost INH; IMB=imatinib PO; INS=insulin; IRO=iron PO; LEV=levabuterol INH; LIS=lisinopril PO; LOR=loratadin PO; LOS=losartan PO; LOV=lovastatin PO; MED=medodrine; MEF=metformin PO; MET=metoprolol PO; $\mathrm{MMF}=$ mycophenolat- mofetil PO; MOM=mometasone PO; MON=montelukast inhal.; $\mathrm{MTX}=$ methotrexate $\mathrm{PO}$; $\mathrm{MTZ}=$ metronidazole $\mathrm{PO}$; NIF=nifedipine; $\mathrm{NOR}=$ norethindrone PO;

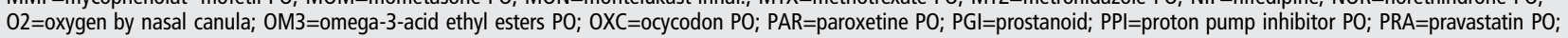

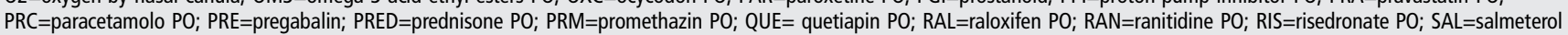
INH; SD=PH study drug; SER=sertraline PO; SIL=sildenafil PO; SIM=simvastatine PO; SIT=sitagliptin PO; SOL=solifenacinsuccinat PO; SPI=spironolactone PO; TAD=tadalafil PO;

TEM=temazepam PO; THY=levothyroxine PO; TOP=topiramat PO; TOR=torsemide PO; TRA=trazodone; TRC=triamcinolon PO; TRAM=tramadole; TREP=trepostinil; TREPIV=trepostinil IV; TREPIN=trepostinil inhal.; TREPPO=trepostinil PO ; TREPSC=trepostinil subcutaneously; TRI=triamterene PO; URS=ursodiol PO; VAL=valsartan PO; VEN=venlaxafine PO; VYT=ezetimibe/ simvastatin PO; WAR=warfarin PO; ZOL=zolpidem PO

tClinical Classification of Pulmonary Hypertension (World Symposium on PH; Nice 2013) as described in Simonneau G, J Am Coll Cardiol 2013. Parenteral prostacyclin administration (intravenous, inhaled, subcutaneous) in the far right column is indicated in bold letters. The definition of WHO functional class of PAH can be found in Rubin LJ. Diagnosis and management of pulmonary arterial hypertension: ACCP Evidence-Based Clinical Practice Guidelines. Introduction. Chest 2004;126:7S-10S.

BMI, body mass index; CTD, PAH associated with connective tissue disease (WSPH Nice PH category 1.4.1); DTI, PAH drug and toxin induced (Nice PH category 1.3); HPAH, heritable PAH (Nice PH category 1.2.); IPAH, idiopathic PAH (Nice PH category 1.1); IV, intravenous; PAH, pulmonary arterial hypertension; PO, oral; SC, subcutaneous; SSRI, selective serotonin reuptake inhibitor. 
Figure 1 Galectin-3 (Gal-3), aldosterone, and N-terminal pro-brain natriuretic peptide (NT-proBNP) plasma concentrations are elevated in pulmonary arterial hypertension (PAH) patients versus controls. The scatter plots on the left and the box and whiskers plots on the right provide data for aldosterone (A and $B)$, galectin-3 (Gal-3) (C and D), and NT-proBNP (E and F) plasma concentrations in control subjects $(n=8)$, patients with idiopathic pulmonary hypertension (IPAH, $n=41$ ), and PAH associated with connective tissue disease (CTD, $n=16)$. The scatter plots show the mean \pm SEM; the box and whiskers plots show the median with IQR $\pm 10-90$ th centile. * $p<0.05$, ${ }^{* *} p<0.01$.
A

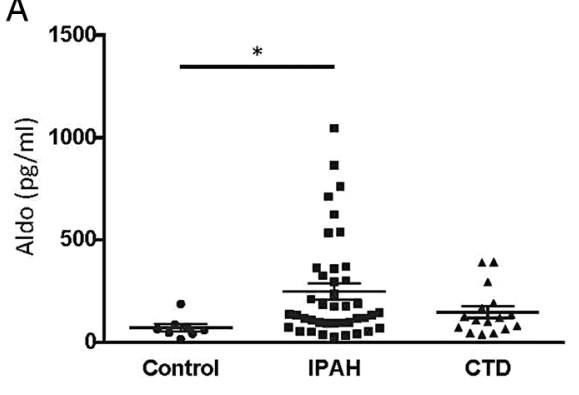

C

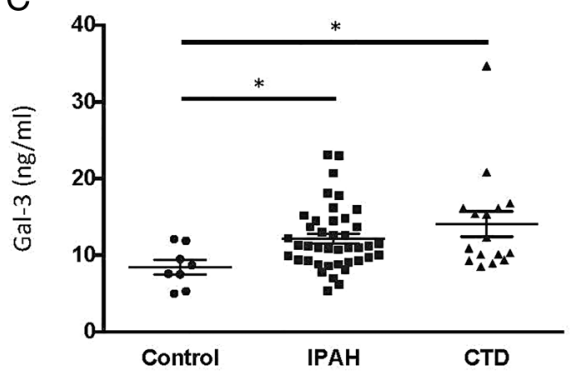

E

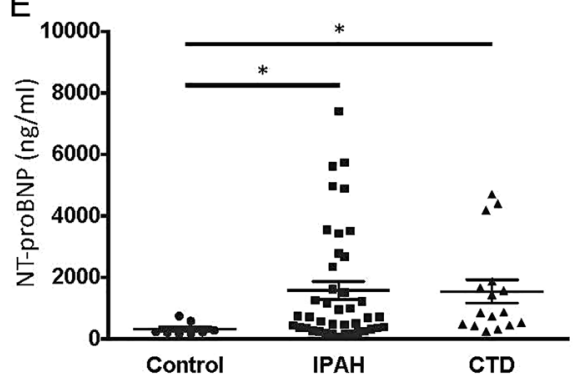

B

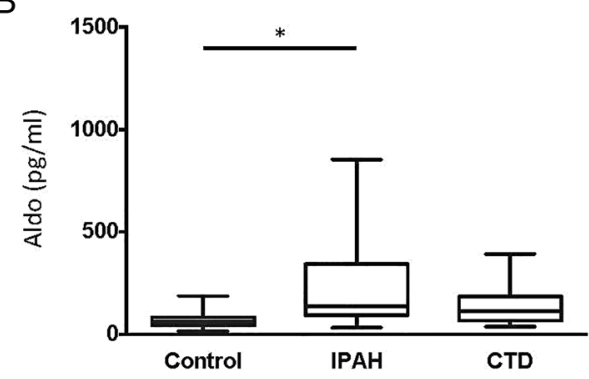

D

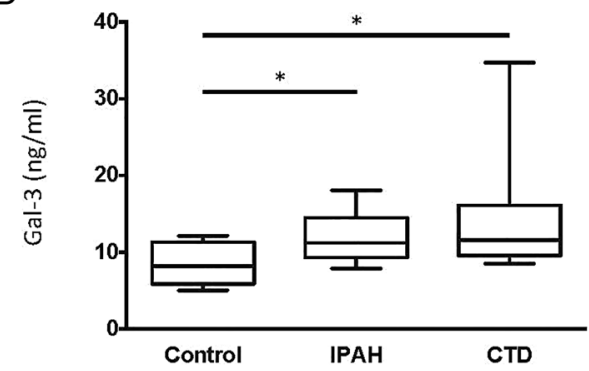

$\mathrm{F}$

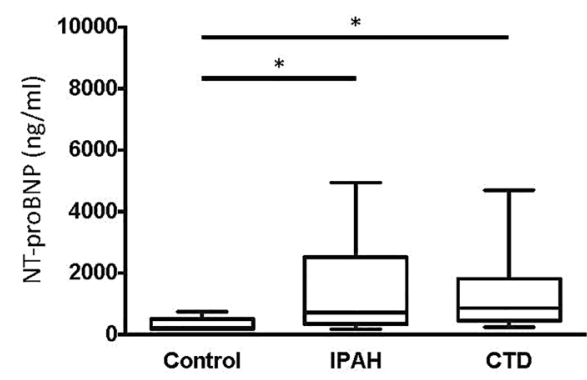

previously been shown to be involved in the pathobiology of $\mathrm{PAH},{ }^{18}$ also showed a trend toward increased plasma concentrations in IPAH patients versus controls (ns; figure $3 \mathrm{~F}$ ). For the other protein biomarkers measured in $\mathrm{PAH}$ and control plasma (IL-16, VEGF, VEGF-C, VEGF-D), no statistically significant differences or clear trends were found (figure 4).

\section{DISCUSSION}

Biomarkers hold the potential to provide valuable insights into the pathobiology and disease progression of $\mathrm{PAH}-\mathrm{a}$ heterogeneous, fatal disease leading to pressure overload and ultimately failure of the right ventricle. Prior biomarker studies identified several plasma proteins being altered in PAH. ${ }^{19}$ However, none of these molecules exhibited all characteristics of an ideal biomarker (eg, high specificity and sensitivity, correlation with disease progression and therapy, etc). Thus, a multiparameter approach has been proposed to adequately reflect the complex and diverse pathogenetic mechanisms of clinical PAH. ${ }^{6}$

This is the first report to show that both plasma aldosterone and Gal-3 are increased in PAH, associated with WHO functional class, thus pointing to the potential of aldosterone/Gal-3 to indicate disease severity. Gal-3 is involved in several pathological and physiological processes-for example, the regulation of organ development, immune reactions, tumorigenesis, tumour growth, and metastasis. ${ }^{20}$ In the heart, Gal-3 augments fibrosis and modulates immune responses, which confers to maladaptive cardiac remodelling. ${ }^{21}$ Extracellular matrix remodelling is a key component of fibrosis, and increased Gal-3, which is released by macrophages, is likely to reflect profibrotic activity. ${ }^{21}$ Since advanced pulmonary hypertensive vascular disease is characterised by the development of vascular and cardiac fibrosis, increased Gal-3 concentrations in PAH may reflect vascular and right ventricular fibrosis. ${ }^{22}$

Gal-3 is activated by aldosterone in vascular smooth muscle cells of the systemic circulation. ${ }^{13}$ Here, we demonstrate that aldosterone is significantly increased in IPAH, but not in PAH-CTD patients, indicating the involvement of aldosterone-independent pathways in CTD, for example, inflammation-mediated induction of Gal-3, possibly mediated by VCAM-1 and IL-12 (figure 2). ${ }^{20}$ Independently of the aldosterone pathway, Gal-3 is expressed by activated macrophages and endothelial cells, attracting inflammatory cells and inducing fibrosis. ${ }^{20}$ This aldosterone-independent Gal-3 secretion might be particularly active in PAH-CTD patients. Interestingly, we did not find a direct correlation between plasma Gal-3 and aldosterone in IPAH patients. Therefore, one might speculate that aldosterone-independent pathways are also relevant in IPAH-associated Gal-3 upregulation. However, we provide data from a single time point, so that further studies addressing the temporal courses of aldosterone and Gal-3 values, and their possible correlation, are needed. Further limitations of this study include uncontrolled dietary and volume status at the time of plasma acquisition.

Both aldosterone and Gal-3 correlated with advanced disease states (as indicated by WHO-FC). It is therefore likely that longitudinal assessments of aldosterone and Gal-3 may help identify patients with more advanced $\mathrm{PAH}$. Aldosterone can be 
Figure 2 Galectin-3 (Gal-3), aldosterone, and N-terminal pro-brain natriuretic peptide (NT-proBNP) plasma concentrations increase with pulmonary arterial hypertension (PAH) severity. The scatter plots on the left and the box and whiskers plots on the right provide data for aldosterone ( $A$ and $B$ ), galectin-3 (Gal-3) (C and D), and NT-proBNP (E and F) plasma concentrations according to WHO functional classes (FC). The scatter plots show the mean $\pm S E M$; the box and whiskers plots show the median with IQR $\pm 10-90$ th centile. Control, $n=8 ; F C ~ I, n=10 ; F C ~ I I+I I I, n=53$. ${ }^{*} \mathrm{p}<0.05,{ }^{* *} \mathrm{p}<0.01$.

Figure 3 Selected potential biomarker plasma concentrations in pulmonary arterial hypertension (PAH) patients. The scatter plots provide data for intercellular adhesion molecule 1 (ICAM-1) (A), vascular cell adhesion molecule 1 (VCAM-1) (B), serum amyloid $A(S A A)(C), C$ reactive protein (CRP) (D), interleukin-12 (IL-12) (E), and fibroblast growth factor 2 (FGF-2) (E) plasma concentrations in control subjects $(n=5)$, patients with idiopathic pulmonary hypertension (IPAH, $n=41$ ), and connective tissue disease-associated pulmonary hypertension (CTD, $n=16$ ). Mean $\pm S E M$; ${ }^{*} p<0.05,{ }^{* *} p<0.01,{ }^{* * * *} p<0.0001$.
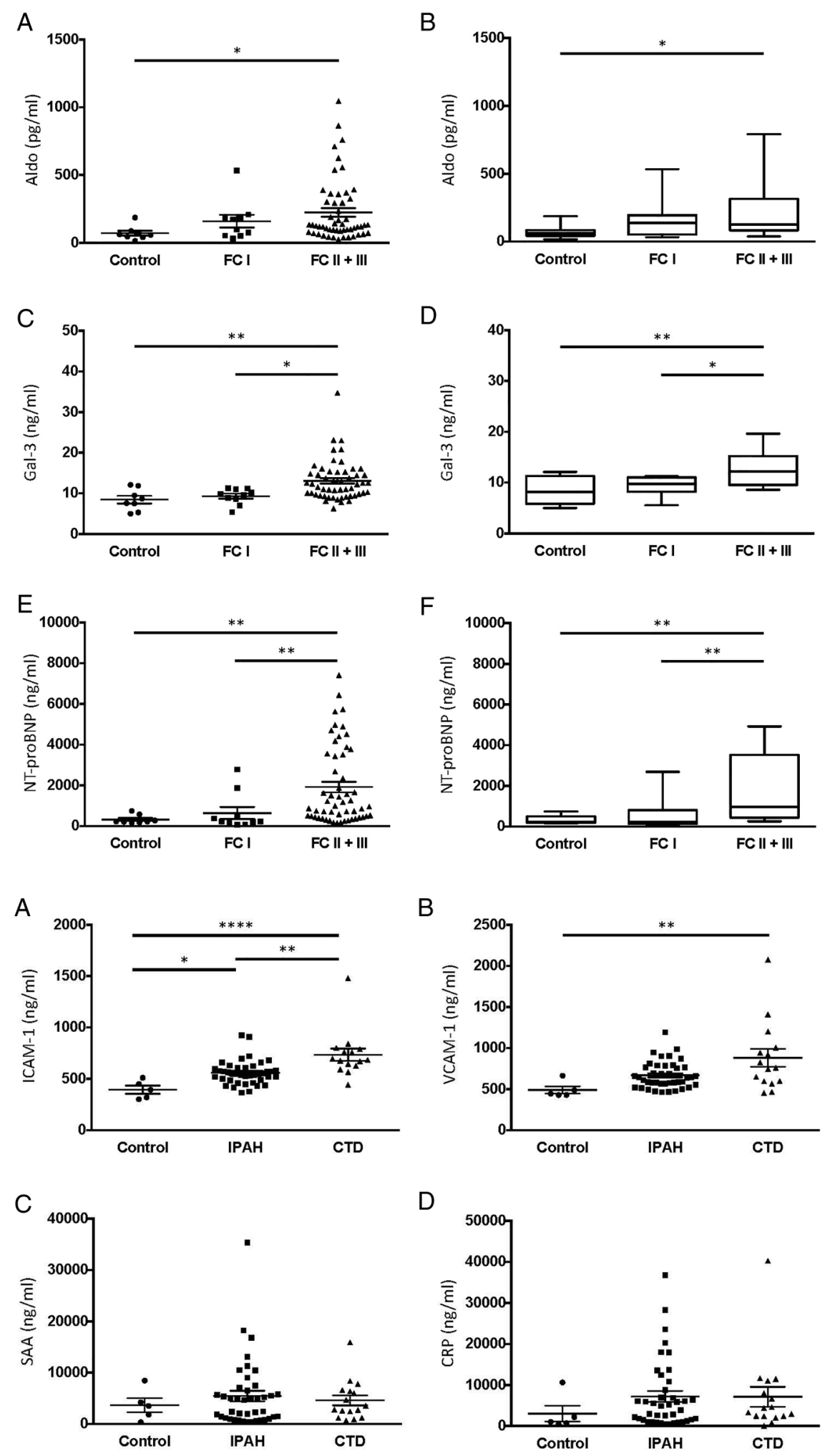

D
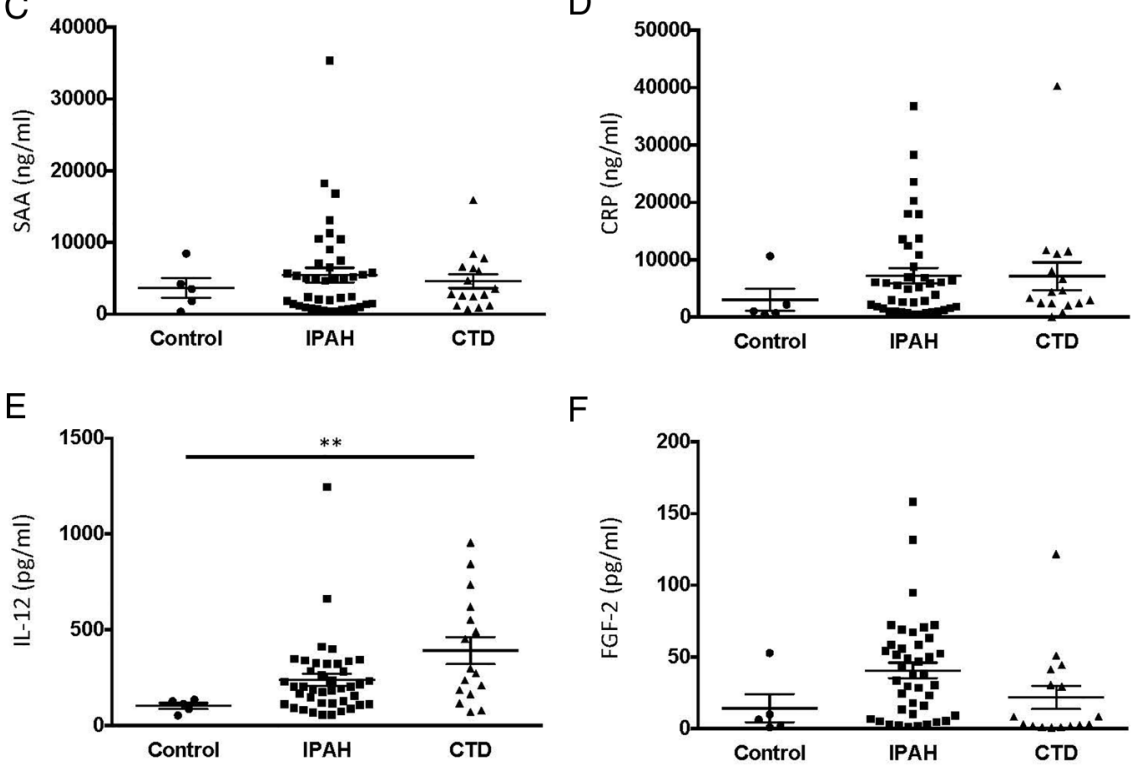
Figure 4 Selected potential biomarker concentrations in pulmonary arterial hypertension (PAH) patients. The scatter plots provide expression level data for interleukin-16 (IL-6) (A), vascular endothelial growth factor (VEGF) (B), VEGF-C (C), and VEGF-D (D) in control subjects $(n=5)$, patients with idiopathic pulmonary hypertension (IPAH, $\mathrm{n}=41$ ), and connective tissue disease-associated pulmonary hypertension (CTD, $n=17$ ).
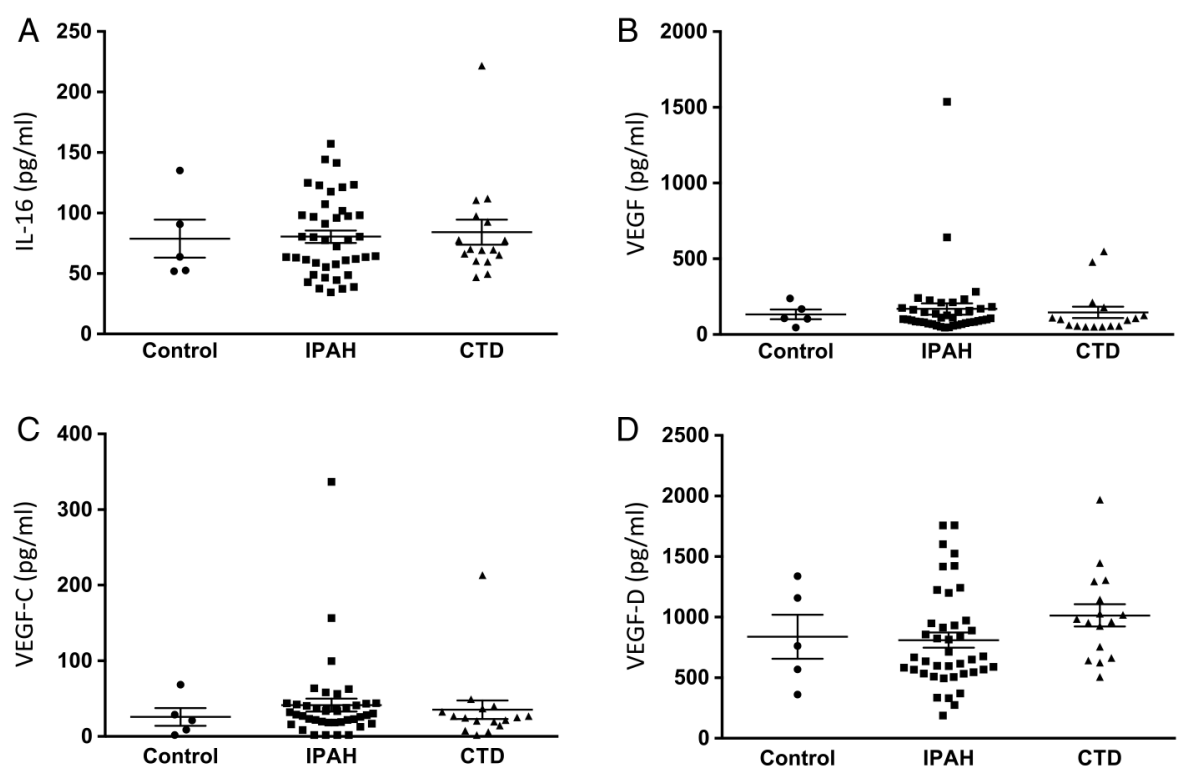

targeted by well-established MR antagonists such as spironolactone, which raises the possibility of treating selected patients with aldosterone antagonists. ${ }^{10} 23$ In fact, cardiac remodelling and dysfunction appears to be responsive to MR blockade (spironolactone) treatment in clinical studies, such as the ARIES and TOPCAT trials. $^{11} 24$

Because plasma aldosterone is not increased in PAH-CTD, while Gal-3 upregulation is observed in both IPAH and PAH-CTD, PAH patients might benefit from direct Gal-3 blockade. These issues therefore need to be addressed in future studies.

Beyond the aldosterone/Gal-3 tandem, we found that ICAM-1 was elevated both in IPAH and PAH-CTD versus controls. Interestingly, in our study, increased IL-12 and VCAM-1 concentrations were only found in PAH-CTD but not in IPAH patients, which indicates PAH-subtype specific roles for these molecules, probably related to the degree of inflammation, vascular injury and anti-angiogenesis. In serum from PAH patients, IL-12 expression values were found to be moderately elevated in IPAH and strongly elevated in PAH-CTD. ${ }^{25}$ In contrast, patients with chronic thromboembolic pulmonary hypertension, who do not show perivascular infiltration of $\mathrm{T}$ and $\mathrm{B}$ lymphocytes in the lungs, had no significant changes in the circulating concentrations of IL-12, indicating that IL-12 elevation is not a direct response to elevated pulmonary arterial pressure. ${ }^{25}$ The association of circulating VCAM-1 and ICAM-1 with PH was also reported in patients with sickle cell disease. ${ }^{26}$ Knockdown of BMPR-II increased ICAM-1 and VCAM-1 expression in HUVECs (human umbilical vein endothelial cells). ${ }^{27}$ These data suggest that VCAM-1 and ICAM-1 induction reflects endothelial activation and dysfunction occurring in $\mathrm{PAH}$.

Taken together, we provide evidence for an involvement of Gal-3 and aldosterone in the pathobiology of human PAH and right ventricular dysfunction, and suggest their potential application as a new tandem in clinical PAH. This study provides the rationale for further clinical and experimental studies investigating the aldosterone/Gal-3 axis in the pathogenesis of PAH. Larger prospective studies including hemodynamics are needed to assess the suitability of aldosterone/Gal-3 as tandem biomarkers for disease progression and treatment response in different $\mathrm{PAH}$ aetiologies.

\section{Key messages}

What is already known on this subject?

The mineralocorticoid receptor agonist aldosterone is involved in pulmonary hypertensive vascular disease and cardiovascular fibrosis. We have previously demonstrated in animal models and in vitro that galectin-3 (Gal-3) is a key mediator of cardiac and renal fibrosis induced by aldosterone.

What might this study add?

This study demonstrates for the first time that the axis aldosterone/galectin-3 (Gal-3) is relevant in pulmonary arterial hypertension (PAH), by showing that plasma levels of both are associated with PAH severity.

How might this impact on clinical practice? Galectin-3 (Gal-3) is already established as a biomarker for cardiac fibrosis, and chronic left heart failure. This work suggest potential applications for aldosterone/galectin-3 (Gal-3) as a new tandem biomarker for clinical PAH.

\section{Author affiliations}

${ }^{1}$ Department of Pediatric Cardiology and Critical Care, Hannover Medical School, Hannover, Germany

${ }^{2}$ Department of Neonatology, Charité University Medical Center, Berlin, Germany ${ }^{3}$ Department of Chemical Engineering, Northeastern University, Boston, Massachusetts, USA

${ }^{4}$ Institute of Clinical Pharmacology, Hannover Medical School, Hannover, Germany ${ }^{5}$ Department of Cardiology and Angiology, Hannover Medical School, Hannover, Germany

${ }^{6}$ Barnett Institute of Chemical and Biological Analysis, Northeastern University, Boston, Massachusetts, USA

Acknowledgements We thank Dr Paolo Galuppo for his advice on the aldosterone ELISA.

Contributors GH conceived the study, and obtained IRB approval, samples and written consent. AH, BDP, HS and SKM participated in the study design, IRB approval and sample collection. LC, EL, and LG performed the biochemical assays and statistical analysis. $L G, H S$, and $G H$ structured and analysed the clinical data sets. GH and HS wrote the paper. HS, LC, EL, JB and GH edited the manuscript.

Funding $\mathrm{GH}$ currently receives grant support from the German Research Foundation (DFG; HA 4348/2-1), Kinderherzen e.V (W-H-001-2014), and Stiftung KinderHerz 
(2511-6-13). LC is the recipient of a postdoctoral research stipend from the European Section of the Aldosterone Council (ESAC). SKM was supported by grant R01 EB009327 from the US National Institutes of Health. The contribution of the COST Action ADMIRE BM1301 is acknowledged (JB).

Competing interests None declared.

Patient consent Obtained.

Ethics approval Northeastern University, Boston (IRB \# 11-07-19).

Provenance and peer review Not commissioned; externally peer reviewed.

\section{REFERENCES}

1 Tuder RM, Archer SL, Dorfmüller P, et al. Relevant issues in the pathology and pathobiology of pulmonary hypertension. J Am Coll Cardiol 2013;62:D4-12.

2 Galiè N, Humbert M, Vachiery JL, et al. 2015 ESC/ERS Guidelines for the diagnosis and treatment of pulmonary hypertension: The Joint Task Force for the Diagnosis and Treatment of Pulmonary Hypertension of the European Society of Cardiology (ESC) and the European Respiratory Society (ERS). Endorsed by: Association for European Paediatric and Congenital Cardiology (AEPC), International Society for Heart and Lung Transplantation (ISHLT). Eur Heart J 2015 Aug 29. pii: ehv317.

3 Abman SH, Hansmann G, Archer S, et al. Pediatric pulmonary hypertension: guidelines from the American Heart Association and American Thoracic Society. Circulation 2015;132:2037-99.

4 Sutendra G, Michelakis ED. Pulmonary arterial hypertension: challenges in translational research and a vision for change. Sci Trans/ Med 2013;5:208sr5.

5 Hansmann G, Plouffe BD, Hatch A, et al. Design and validation of an endothelial progenitor cell capture chip and its application in patients with pulmonary arterial hypertension. J Mol Med 2011;89:971-83.

6 Pezzuto B, Badagliacca R, Poscia R, et al. Circulating biomarkers in pulmonary arterial hypertension: update and future direction. J Heart Lung Transplant 2015;34:282-305

7 Maron BA, Leopold JA. The role of the renin-angiotensin-aldosterone system in the pathobiology of pulmonary arterial hypertension (2013 Grover Conference series). Pulm Circ 2014;4:200-10

8 Maron BA, Opotowsky AR, Landzberg MJ, et al. Plasma aldosterone levels are elevated in patients with pulmonary arterial hypertension in the absence of left ventricular heart failure: a pilot study. Eur J Heart Fail 2013;15:277-83.

9 Maron BA, Zhang $Y-Y$, White $K$, et al. Aldosterone inactivates the endothelin-B receptor via a cysteinyl thiol redox switch to decrease pulmonary endothelial nitric oxide levels and modulate pulmonary arterial hypertension. Circulation 2012;126:963-74.

10 Maron BA, Leopold JA. Emerging concepts in the molecular basis of pulmonary arterial hypertension: part II: neurohormonal signaling contributes to the pulmonary vascular and right ventricular pathophenotype of pulmonary arterial hypertension. Circulation 2015:131:2079-91.

11 Maron BA, Waxman AB, Opotowsky AR, et al. Effectiveness of spironolactone plus ambrisentan for treatment of pulmonary arterial hypertension (from the [ARIES] study 1 and 2 trials). Am J Cardiol 2013;112:720-5.
12 Bauersachs J, Jaisser F, Toto R. Mineralocorticoid receptor activation and mineralocorticoid receptor antagonist treatment in cardiac and renal diseases. Hypertension 2015;65:257-63.

13 Calvier L, Miana M, Reboul P, et al. Galectin-3 mediates aldosterone-induced vascular fibrosis. Arterioscler Thromb Vasc Biol 2013;33:67-75.

14 Calvier L, Martinez-Martinez E, Miana M, et al. The impact of galectin-3 inhibition on aldosterone-induced cardiac and renal injuries. JACC Heart Fail 2015;3:59-67.

15 Edelmann F, Wachter R, Schmidt AG, et al. Effect of spironolactone on diastolic function and exercise capacity in patients with heart failure with preserved ejection fraction: the Aldo-DHF randomized controlled trial. JAMA 2013;309:781-91.

16 Edelmann F, Holzendorf V, Wachter $\mathrm{R}$, et al. Galectin-3 in patients with heart failure with preserved ejection fraction: results from the Aldo-DHF trial. Eur J Heart Fail 2015:17:214-23.

17 Yancy CW, Jessup M, Bozkurt B, et al. 2013 ACCF/AHA guideline for the management of heart failure: a report of the American College of Cardiology Foundation/American Heart Association Task Force on Practice Guidelines. J Am Coll Cardiol 2013:62:e147-239.

18 Izikki M, Guignabert C, Fadel E, et al. Endothelial-derived FGF2 contributes to the progression of pulmonary hypertension in humans and rodents. J Clin Invest 2009;119:512-23.

19 Cracowski J-L, Leuchte $\mathrm{HH}$. The potential of biomarkers in pulmonary arterial hypertension. Am J Cardiol 2012;110:32S-8S.

20 Gruson D, Ko G. Galectins testing: new promises for the diagnosis and risk stratification of chronic diseases? Clin Biochem 2012:45:719-26.

21 Sharma UC, Pokharel S, van Brakel TJ, et al. Galectin-3 marks activated macrophages in failure-prone hypertrophied hearts and contributes to cardiac dysfunction. Circulation 2004;110:3121-8.

22 Nishi $Y$, Sano $H$, Kawashima $T$, et al. Role of galectin-3 in human pulmonary fibrosis. Allergol Int 2007;56:57-65.

23 Azibani F, Fazal L, Chatziantoniou C, et al. Aldosterone mediates cardiac fibrosis in the setting of hypertension. Curr Hypertens Rep 2013;15:395-400.

24 Shah AM, Shah SJ, Anand IS, et al. Cardiac structure and function in heart failure with preserved ejection fraction: baseline findings from the echocardiographic study of the Treatment of Preserved Cardiac Function Heart Failure with an Aldosterone Antagonist trial. Circ Heart Fail 2014;7:104-15.

25 Larsen KO, Yndestad A, Sjaastad I, et al. Lack of CCR7 induces pulmonary hypertension involving perivascular leukocyte infiltration and inflammation. Am J Physiol Lung Cell Mol Physiol 2011;301:L50-9.

26 Kato GJ, Martyr S, Blackwelder WC, et al. Levels of soluble endothelium-derived adhesion molecules in patients with sickle cell disease are associated with pulmonary hypertension, organ dysfunction, and mortality. Br J Haematol 2005; 130:943-53.

27 Kim CW, Song H, Kumar S, et al. Anti-inflammatory and antiatherogenic role of BMP receptor II in endothelial cells. Arterioscler Thromb Vasc Biol 2013;33:1350-9 